\title{
Faces from Sketches: A Subspace Synthesis Approach
}

\author{
Yung-hui Li ${ }^{\mathrm{a}}$, Marios Savvides ${ }^{\mathrm{b}}$ \\ ${ }^{a}$ Language Technology Institute, Carnegie Mellon University, 407 S. Craig St, Room 207, \\ Pittsburgh, PA 15213; \\ ${ }^{\mathrm{b}}$ Dept of Electrical and Computer Engineering, Carnegie Mellon University, 5000 Forbes Ave, \\ Pittsburgh, PA 15213
}

\begin{abstract}
In real life scenario, we may be interested in face recognition for identification purpose when we only got sketch of the face images, for example, when police tries to identify criminals based on sketches of suspect, which is drawn by artists according to description of witnesses, what they have in hand is a sketch of suspects, and many real face image acquired from video surveillance. So far the state-of-the-art approach toward this problem tries to transform all real face images into sketches and perform recognition on sketch domain. In our approach we propose the opposite which is a better approach; we propose to generate a realistic face image from the composite sketch using a Hybrid subspace method and then build an illumination tolerant correlation filter which can recognize the person under different illumination variations. We show experimental results on our approach on the CMU PIE (Pose Illumination and Expression) database on the effectiveness of our novel approach.
\end{abstract}

Keywords: face recognition, correlation filter, face-sketch hybrid subspace, pattern recognition, computer vision, security

\section{INTRODUCTION}

Automatic criminal identification and tracking is a very interesting topic in terms of both research and developing practical system that can be deployed in the field. In many criminal investigations there are usually one or two human witnesses that have caught a glimpse of the criminal or terrorist suspects and the police and government authorities must use this vital information as means to catch these suspects. In such cases, typically a professional police sketch artist will work and co-operate with the witnesses to develop and synthesize a police sketch of the suspect. This sketch is then distributed among police officers in efforts to look for the suspects at ports of entry or other locations. The quality of the reconstructed sketch is extremely important for the officers as they visually have to recognize and find the suspect.

To summarize, we can describe the problem we want to solve as following:

- Available training data: a collection of face images which are recording by video surveillance, hence, those face images may suffer from great illumination variation.

- Probe image: a sketch of face, drawn by artist based on description of witnesses of crime scene.

- Objective: identify who this person is based on the probe sketch and our database

\subsection{Previous work}

X. Tang and X. Wang have done fabulous work toward this sketch-face reconstruction and recognition problem ${ }^{1 \sim 4}$. They attack this problem by dividing the work into two phases: (1) Photo-to-Sketch Transformation (2) Recognition on sketch space. Since their approach is symmetric and reversible, one can also do the experiment the other way around, i.e: first transform the sketch image into real face image then perform recognition in face space.

Following the standard eigenface approach ${ }^{5}$, they compute eigenvector matrix $U_{p}$ for face images and the eigenvector matrix $U_{s}$ for sketch images. For a new face image $P_{f}$, after subtracting the mean of all face images, one can easily project it into face eigenspace and get a coefficient $b_{p}$ for reconstruction in eigenface domain. Then they synthesize sketch of this face image by taking product of $U_{s}$ and $b_{p}$, then adding the mean of all sketch image. 
For recognition, with each test face image, they first perform face-to-sketch transformation, described above, then they calculate the distance of the projection coefficients between the synthesized sketch image and each of sketch image in training data to identify which class this synthesized image belonged to. Of course, one can do the task the other way around, i.e, given a test sketch image, one can perform sketch-to-face transformation (which is similar to face-to-sketch transformation, just in reverse direction) first, then calculate the distance of the projection coefficients between the synthesized face image and each face image in training data. They compared these two approaches in their paper.

\section{METHODOLOGY}

We propose a new method to attack the problem. At first stage (Transformation Phase), unlike Tang and Wang's approach, which performs eigenface method within two different spaces (face and sketch), we propose to combine the image vector from both face and sketch to form a hybrid space, and do eigenface analysis on this hybrid space. The eigenvector calculated from this hybrid space is supposed to capture the orthonormal basis vector across this hybrid space, which should reveal more information about interdependency between face image and corresponding sketch image. Theoretically speaking, our proposed hybrid method should perform not worse than Tang and Wang's method and should outperform their result when our database is big enough.

At second stage, instead of using distance-based classifiers, we propose to use an illumination tolerant, frequency-based technique, which has been tested and proved to be a very useful and powerful patter recognition technique ${ }^{6 \sim 8}$. It's advanced correlation filter. Let's divided our approaches into three stages: (1) Training Stage (2) Synthesis Stage and (3) Recognition Stage. We will explain more details in each stage in following sub-sections.

\subsection{Training Stage}

What we are trying to do in training stage is to build a model for both face and sketch images so that later in Synthesis Stage we can use these models to synthesize face image when we only have sketch image. In this stage, the data we have already is a collection of face images, perhaps recorded by video surveillance. In order to achieve our goal, we should (1) get a collection of sketch images, each of which is a sketch of one face image in our face database; (2) make use of some powerful machine learning technique to discover and preserve the statistical dependency between our face and sketch database, and save it as our model, which can be used in Synthesis Stage.

For task (1), since we don't have corresponding sketch image in our database, we use Adobe Photoshop 7.0 to manually transform each face image into their sketch counterpart. The function is in menu Filter $->$ Sketch $->$ Photocopy. We use exactly the same parameter when transforming each image to ensure the transformation process preserves the same information for each image. Examples of images of face-sketch pairs are shown in Fig 1.
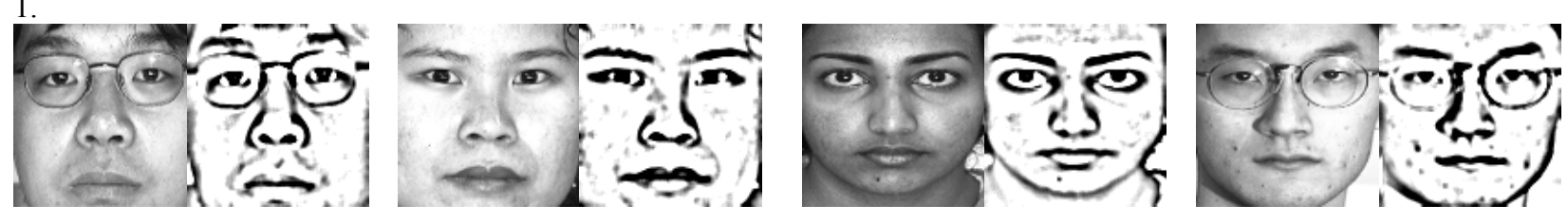

Figure 1: examples of face images and their sketch counterparts.

As for Task (2), Principle Component Analysis (PCA) or Eigenface approach has been widely used in face recognition problem 9 . Eigenface approach calculates and preserves the principle components (PCs) of data and these PCs can be used to reconstruct a new face image later. But now our problem contains two types of data which is implicitly interdependent with each other. How are we going to use eigenface method to deal with our problem?

One naïve approach is to do eigen-analysis on face and sketch domain independently and get eigenface and eigensketch, respectively. When one probe sketch image comes in, get projection coefficients by projecting this sketch onto eigensketch space. Finally, by using these projection coefficients as weighting factors on eigenface domain, one can synthesize the original face image. This is the main approach of the work of Tang and Wang ${ }^{3}$. 
Basically, this approach neglects the interdependency between eigenface and eigensketch during derivation, and later assumes there's a strong dependency between eigenface and eigensketch by saying that they can share the same projection coefficients. In contrast with this approach, we argue that we should take into consideration the interdependency between eigenface and eigensketch during derivation so that derived eigenface and eigensketch maintain their correlation between each other.

Suppose we have a set of real face image $F_{1}, F_{2}, \ldots F_{m}$, and a set of sketch images $S_{1}, S_{2}, \ldots S_{m}$, where $S_{i}$ is the sketch image of $F_{i}$. First we compute the mean of $F_{i}$ and $S_{i}$ to get $m_{F}$ and $m_{S}$, ie: $m_{F}=\left(F_{1}+F_{2}+\ldots F_{m}\right) / m$; $\mathrm{m}_{\mathrm{S}}=\left(\mathrm{S}_{1}+\mathrm{S}_{2}+\ldots \mathrm{S}_{\mathrm{m}}\right) / \mathrm{m}$. Then we subtract the mean from each image to get $\mathrm{B}_{\mathrm{i}}$ and $\mathrm{D}_{\mathrm{i}}$, i.e: $\mathrm{B}_{\mathrm{i}}=\mathrm{F}_{\mathrm{i}}-\mathrm{m}_{\mathrm{F}}, \mathrm{D}_{\mathrm{i}}=\mathrm{S}_{\mathrm{i}}-\mathrm{m}_{\mathrm{S}}$

Then we form a matrix $X_{h}=\left[P_{1}, P_{2}, \ldots P_{m}\right]$ where $P_{i}$ is a column vector derived from concatenating $B_{i}$ and $D_{i}$, i.e: we form a hybrid space by concatenating the variance of face space and sketch space. After that, we follow the standard method of eigenface method to derive the orthonormal eigenvector matrix of the covariance matrix $\mathrm{W}$. In details: $\mathrm{W}=$ $\mathrm{X}_{\mathrm{h}} \mathrm{X}_{\mathrm{h}}{ }^{\mathrm{T}}$ is the covariance matrix in hybrid space. We want to find the principle component of $\mathrm{W}$ in order to capture the direction of maximal variations. In general, direct computation of eigenvectors of $\mathrm{W}$ is not practical since the dimensionality of $\mathrm{W}$ is too large. But since sample images number is in general much smaller than the dimensionality, the rank of $W$ is only $m-1$. So the eigenvector of the smaller matrix $X_{h}{ }^{T} X_{h}$ can be computed as $\left(X_{h}{ }^{T} X_{h}\right) V_{h}=V_{h} D_{h}, w_{h}$,ere $V_{h}$ is the eigenvector matrix and $D_{h}$ is the diagonal eigenvalue matrix. Multiplying both sides by $X_{h}$, we have $\left(\mathrm{X}_{\mathrm{h}} \mathrm{X}_{\mathrm{h}}^{\mathrm{T}}\right) \mathrm{X}_{\mathrm{h}} \mathrm{V}_{\mathrm{h}}=\mathrm{X}_{\mathrm{h}} \mathrm{V}_{\mathrm{h}} \mathrm{D}_{\mathrm{h}}$
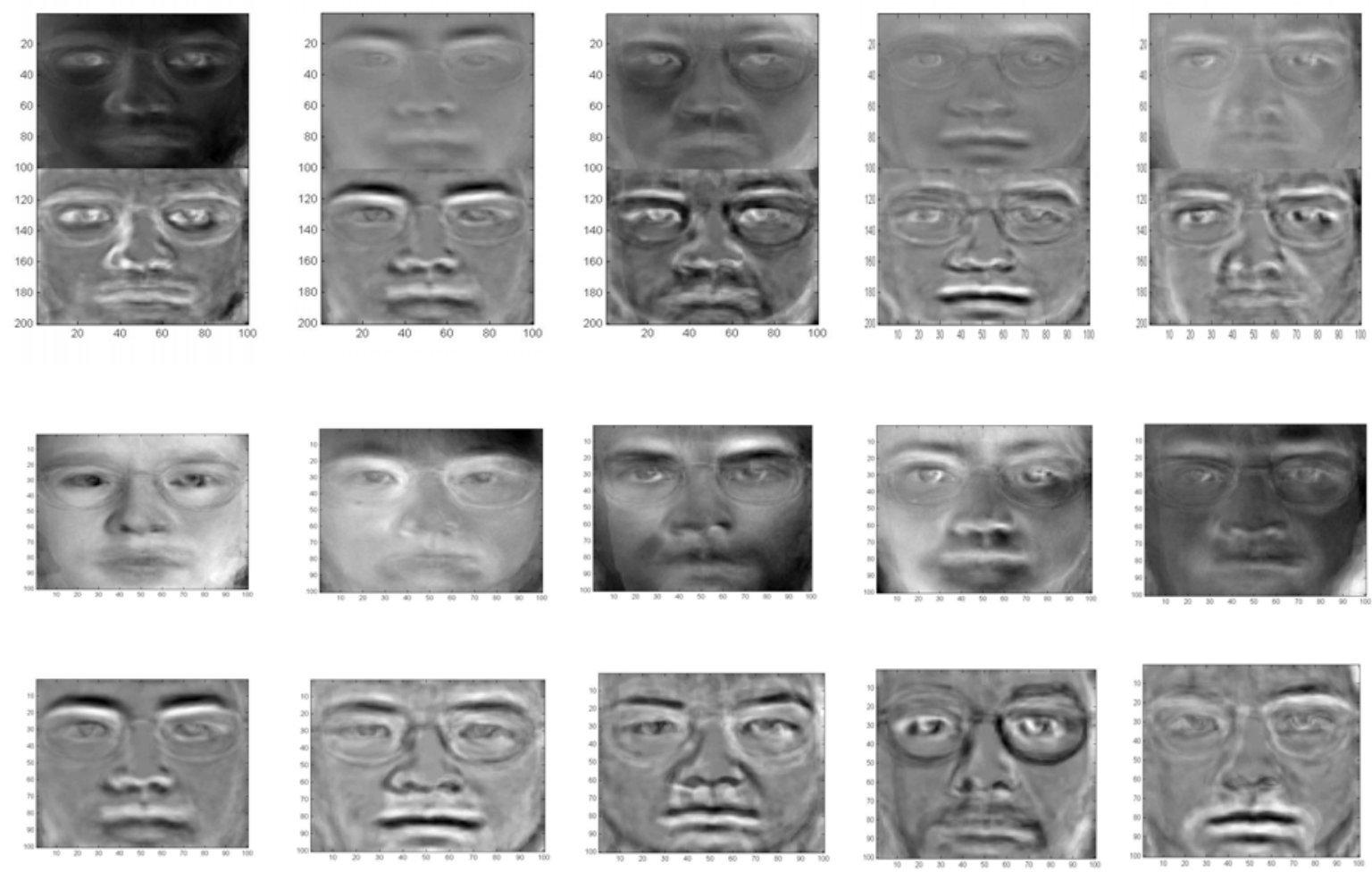

Figure 2: The comparison of eigenfaces derived from three difference spaces. The first row is the eigenfaces derived from hybrid space. The second row is the eigenfaces derived from face images only. The third row is the eigensketch derived from sketch images only. Note that while the pseudo-eigenface and pseudo-eigensketch images are highly correlated among the images at first row, the eigenfaces in second row are not correlated to the eigensketch in third row. Hence, calculating eigenface and eigensketch independently will not keep the corresponding information between each other, while calculating eigenface in hybrid space will. 


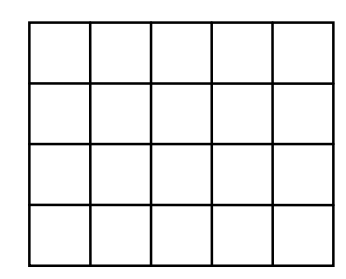

Face images
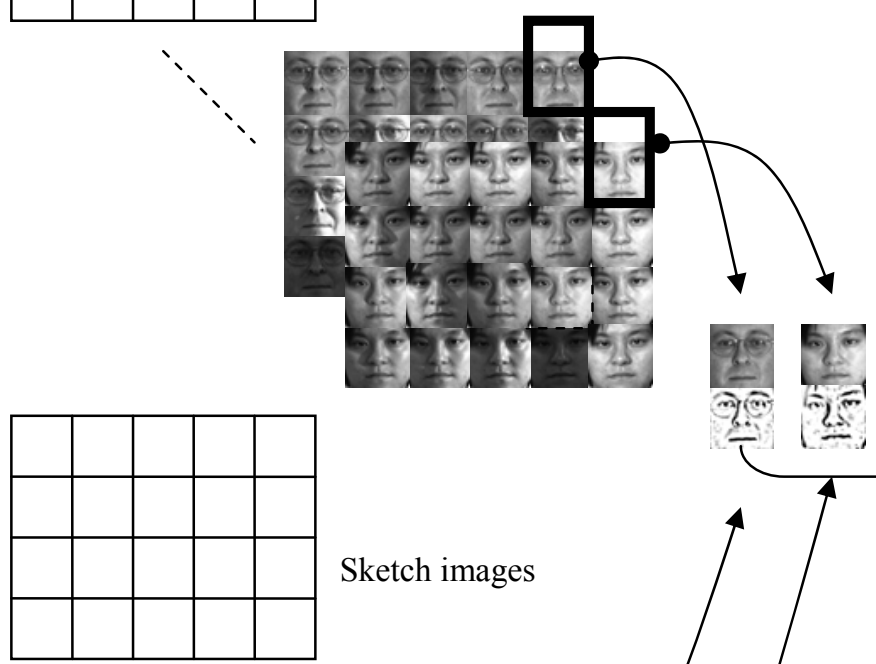

Sketch images

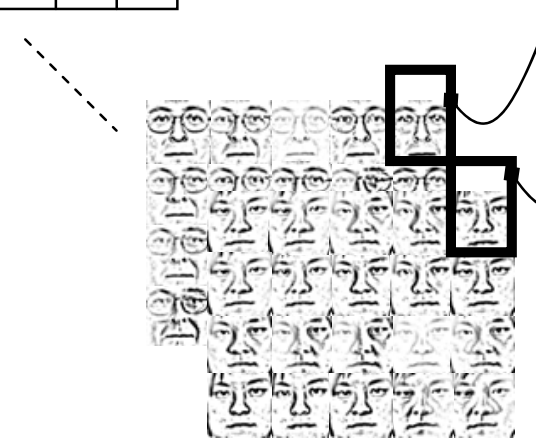

일

- - - (Hybrid Space)

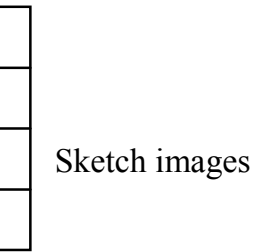

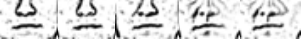
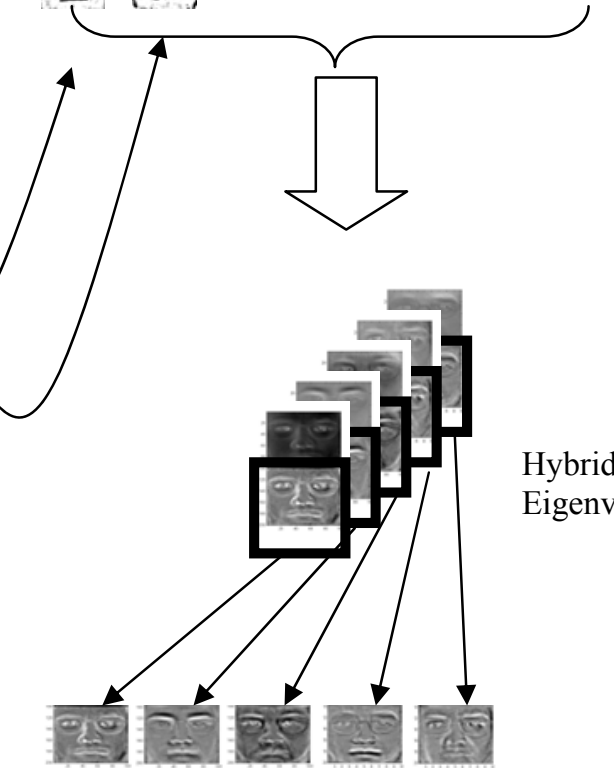

Hybrid

Eigenvectors

Pseudo-eigensketch is the lower half of the hybrid eigenvectors

Figure 3: illustration of experimental procedure during training stage.

Therefore, the orthonormal eigenvector matrix of the covariance matrix $W$ is $U_{h}=X_{h} V_{h}$

Note that this eigenvector matrix $U_{h}$ is for hybrid space. If we plot each column of $U_{h}$ into a $2 D$ gray scale graph, one can see that it contains two images. We can treat it as the upper half image is a "pseudo-eigenvector" for face images (we can call it pseudo-eigenface) and the lower half image is a "pseudo-eigenvector" for sketch images (call it pseudoeigensketch). Examples of pseudo-eigenface and pseudo-eigensketch are shown in Fig 2.

In Fig 2, we can notice an interesting and important fact that if we calculate eigenface and eigensketch independently from our database, the resulting eigenfaces are not correlated to resulting eigensketches. But by performing eigenanalysis on hybrid subspace, we can clearly see that the upper half of each eigenvector is highly correlated with the 
lower half of it, which means our proposed method can capture the nonlinear mapping function between face and sketch images.

Fig. 3 gives us a graphical illustration of our procedure in Training Stage.

\subsection{Synthesis Stage}

In this stage, the problem we want to solve is: given a probe sketch image, with pseudo-eigenface and pseudoeigensketch models at hand, how can we synthesize the original face image?

One can easily think of the standard procedure in eigenface approach, i.e, project the sketch image on eigensketch subspace and get projection coefficients. But now what we have is "pseudo" eigenface and "pseudo" eigensketch. Pseudo-eigenface and Pseudo-eigensketch are not "real" eigenvectors by themselves. They are eigenvectors when being concatenated in hybrid space. Once we split the hybrid space into two parts, the orthogonality is destroyed. So we should not treat them as eigenvectors.

Instead, we use "pseudo-inverse" least squares fitting technique to compute the projection coefficients since this method gives us projections with least-squared errors. After we have projection coefficients (PCs) of the probe sketch image, we use PCs on hybrid eigenvectors to synthesize a new image in hybrid subspace. This new image in hybrid subspace will consist of two parts. The lower half should be an approximation of the probe sketch image, and the upper half should be corresponding original face image. By keeping only the upper half of the image, we finish the process of face image synthesis. The steps we take in Synthesis Stage are illustrated in Fig. 4.

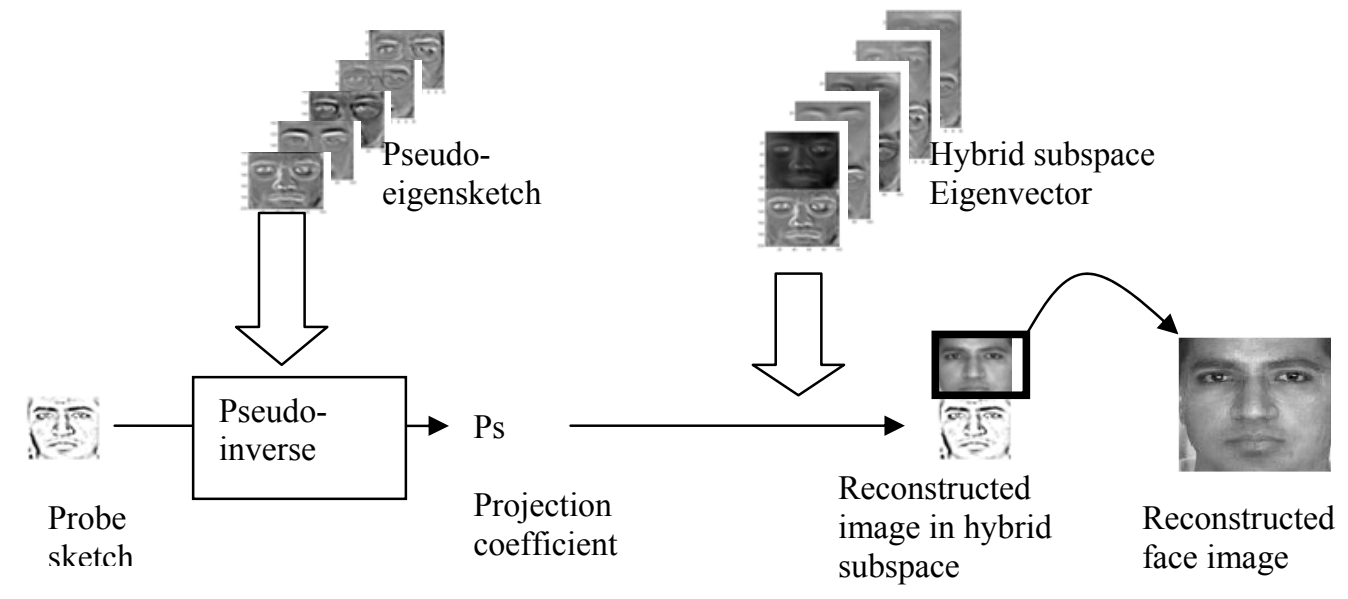

Figure 4: illustration of experimental procedure during reconstruction phase.

\subsection{Mathematical Justification of our approach}

We can justify our approach is better than previous work not only from experiment result, but also from insight derived from mathematical equations. Assume $\mathrm{x}_{\mathrm{f}}$ and $\mathrm{x}_{\mathrm{s}}$ denotes the face and sketch data, respectively. When performing eigenanalysis on face domain, we are trying to find the projective direction $\omega_{\mathrm{f}}$ onto which $\mathrm{x}_{\mathrm{f}}$ has maximal variation. Hence, our objective function $\mathrm{J}\left(\omega_{\mathrm{f}}\right)$ is: 


$$
\begin{aligned}
\mathrm{J}\left(\omega_{\mathrm{f}}\right) & =\operatorname{Var}\left(\omega_{\mathrm{f}}{ }^{\mathrm{T}} \mathrm{x}_{\mathrm{f}}\right)=\mathrm{E}\left(\omega_{\mathrm{f}}{ }^{\mathrm{T}} \mathrm{x}_{\mathrm{f}}-\omega_{\mathrm{f}}{ }^{\mathrm{T}} \mu_{\mathrm{f}}\right)^{2}=\mathrm{E}\left(\omega_{\mathrm{f}}{ }^{\mathrm{T}} \mathrm{x}_{\mathrm{f}}-\omega_{\mathrm{f}}{ }^{\mathrm{T}} \mu_{\mathrm{f}}\right)\left(\mathrm{x}_{\mathrm{f}}{ }^{\mathrm{T}} \omega_{\mathrm{f}}-\mu^{\mathrm{T}} \omega_{\mathrm{f}}\right) \\
& =\omega_{\mathrm{f}}{ }^{\mathrm{T}} \mathrm{E}\left(\mathrm{x}_{\mathrm{f}}-\mu_{\mathrm{f}}\right)\left(\mathrm{x}_{\mathrm{f}}-\mu_{\mathrm{f}}\right)^{\mathrm{T}} \omega_{\mathrm{f}}=\omega_{\mathrm{f}}{ }^{\mathrm{T}} \Sigma_{\mathrm{f}} \omega_{\mathrm{f}}
\end{aligned}
$$

where $\mu_{\mathrm{f}}$ is the mean of the face data $\mathrm{x}_{\mathrm{f}}$ and $\Sigma_{\mathrm{f}}=\mathrm{E}\left(\mathrm{x}_{\mathrm{f}}-\mu_{\mathrm{f}}\right)\left(\mathrm{x}_{\mathrm{f}}-\mu_{\mathrm{f}}\right)^{\mathrm{T}}$

So our objective is to maximize $\mathrm{J}\left(\omega_{\mathrm{f}}\right)=\omega_{\mathrm{f}}{ }^{\mathrm{T}} \Sigma_{\mathrm{f}} \omega_{\mathrm{f}}$, subject to the constraint that $\omega_{\mathrm{f}}{ }^{\mathrm{T}} \omega_{\mathrm{f}}=1$. We can solve this problem by Lagrange Multiplier:

$$
\mathrm{L}\left(\omega_{\mathrm{f}}, \lambda\right)=\omega_{\mathrm{f}}^{\mathrm{T}} \Sigma_{\mathrm{f}} \omega_{\mathrm{f}}-\lambda\left(\omega_{\mathrm{f}}^{\mathrm{T}} \omega_{\mathrm{f}}-1\right)
$$

Take derivative with respect to $\omega_{\mathrm{f}}$ and set it to zero:

$$
\begin{aligned}
& \frac{\partial \mathrm{L}\left(\omega_{\mathrm{f}}, \lambda\right)}{\partial \omega_{\mathrm{f}}}=2 \Sigma_{\mathrm{f}} \omega_{\mathrm{f}}-2 \lambda \omega_{\mathrm{f}}=0 \\
& \Sigma_{\mathrm{f}} \omega_{\mathrm{f}}=\lambda_{\mathrm{f}} \omega_{\mathrm{f}}
\end{aligned}
$$

From here we can see that the the projective direction $\omega_{\mathrm{f}}$ we are looking for is indeed the eigenvectors of covariance matrix $\Sigma_{\mathrm{f}}$

Same argument applies to sketch domain. If $\Sigma_{\mathrm{s}}$ is the covariance matrix of sketch data, i.e:

$$
\Sigma_{\mathrm{s}}=\mathrm{E}\left(\mathrm{x}_{\mathrm{s}}-\mu_{\mathrm{s}}\right)\left(\mathrm{x}_{\mathrm{s}}-\mu_{\mathrm{s}}\right)^{\mathrm{T}}
$$

After Lagrange Multiplier method, we have

$$
\Sigma_{\mathrm{s}} \omega_{\mathrm{s}}=\lambda_{\mathrm{s}} \omega_{\mathrm{s}}
$$

Now, let's look at what we will get if we perform eigen-analysis on a hybrid subspace created from concatenating face and sketch data. Suppose $\mathrm{x}_{\mathrm{f}}$ and $\mathrm{x}_{\mathrm{s}}$ denotes the face and sketch data, respectively, as stated above. The vector in hybrid space is formed by:

$$
x_{h}=\left[\frac{x_{f}}{x_{s}}\right]
$$

Then our objective function $\mathrm{J}\left(\omega_{\mathrm{h}}\right)$ will become

$$
\mathrm{J}\left(\omega_{\mathrm{h}}\right)=\omega_{\mathrm{h}}^{\mathrm{T}} \Sigma_{\mathrm{h}} \omega_{\mathrm{h}}
$$

where $\Sigma_{h}=E\left(x_{h}-\mu_{h}\right)\left(x_{h}-\mu_{h}\right)^{T}$, and $\mu_{h}$ is the mean of the hybrid data $x_{h}$

Substitute (6) into $\Sigma_{\mathrm{h}}$ and we will get

$$
\mathrm{x}_{\mathrm{h}}-\mu_{\mathrm{h}}=\left[\frac{\mathrm{x}_{\mathrm{f}}}{\mathrm{x}_{\mathrm{s}}}\right]-\left[\frac{\mu_{\mathrm{f}}}{\mu_{\mathrm{s}}}\right]=\left[\frac{\mathrm{x}_{\mathrm{f}}-\mu_{\mathrm{f}}}{\mathrm{x}_{\mathrm{s}}-\mu_{\mathrm{s}}}\right]
$$




$$
\begin{aligned}
\Sigma_{\mathrm{h}} & =\mathrm{E}\left(\mathrm{x}_{\mathrm{h}}-\mu_{\mathrm{h}}\right)\left(\mathrm{x}_{\mathrm{h}}-\mu_{\mathrm{h}}\right)^{\mathrm{T}}=\mathrm{E}\left\{\left[\frac{\mathrm{x}_{\mathrm{f}}-\mu_{\mathrm{f}}}{\mathrm{x}_{\mathrm{s}}-\mu_{\mathrm{s}}}\right] \bullet\left[\left(\mathrm{x}_{\mathrm{f}}-\mu_{\mathrm{f}}\right)^{\mathrm{T}} \mid\left(\mathrm{x}_{\mathrm{s}}-\mu_{\mathrm{s}}\right)^{\mathrm{T}}\right]\right\} \\
& =\mathrm{E}\left[\frac{\left(\mathrm{x}_{\mathrm{f}}-\mu_{\mathrm{f}}\right)\left(\mathrm{x}_{\mathrm{f}}-\mu_{\mathrm{f}}\right)^{\mathrm{T}}}{\left(\mathrm{x}_{\mathrm{s}}-\mu_{\mathrm{s}}\right)\left(\mathrm{x}_{\mathrm{f}}-\mu_{\mathrm{f}}\right)^{\mathrm{T}}} \mid \frac{\left(\mathrm{x}_{\mathrm{f}}-\mu_{\mathrm{f}}\right)\left(\mathrm{x}_{\mathrm{s}}-\mu_{\mathrm{s}}\right)^{\mathrm{T}}}{\left(\mathrm{x}_{\mathrm{s}}-\mu_{\mathrm{s}}\right)\left(\mathrm{x}_{\mathrm{s}}-\mu_{\mathrm{s}}\right)^{\mathrm{T}}}\right] \\
& =\left[\frac{\sum_{\mathrm{f}}}{\sum_{\mathrm{sf}}} \mid \frac{\sum_{\mathrm{fs}}}{\sum_{\mathrm{s}}}\right]
\end{aligned}
$$

Where $\Sigma_{\mathrm{fs}}=\mathrm{E}\left(\mathrm{x}_{\mathrm{f}}-\mu_{\mathrm{f}}\right)\left(\mathrm{x}_{\mathrm{s}}-\mu_{\mathrm{s}}\right)^{\mathrm{T}}, \quad \Sigma_{\mathrm{sf}}=\mathrm{E}\left(\mathrm{x}_{\mathrm{s}}-\mu_{\mathrm{s}}\right)\left(\mathrm{x}_{\mathrm{f}}-\mu_{\mathrm{f}}\right)^{\mathrm{T}}$

Let $\omega_{\mathrm{sf}}$ and $\omega_{\mathrm{ss}}$ denotes pseudo-eigenface and pseudo-eigensketch in our approach, respectively. In our approach, we calculate $\omega_{\mathrm{sf}}$ and $\omega_{\mathrm{ss}}$ from decomposing the hybrid eigenmatrix $\omega_{\mathrm{h}}$ :

$$
\omega_{\mathrm{h}}=\left[\frac{\omega_{\mathrm{sf}}}{\omega_{\mathrm{ss}}}\right]
$$

The relationship $\Sigma_{\mathrm{h}}$ between and $\omega_{\mathrm{h}}$ is:

$$
\Sigma_{\mathrm{h}} \omega_{\mathrm{h}}=\lambda_{\mathrm{h}} \omega_{\mathrm{h}}
$$

Substitute $\Sigma_{\mathrm{h}}$ and $\omega_{\mathrm{h}}$ with (9) and (10) into (11) we will get:

$$
\begin{aligned}
& \Sigma_{\mathrm{h}} \omega_{\mathrm{h}}=\left[\frac{\Sigma_{\mathrm{f}}}{\sum_{\mathrm{sf}}} \mid \frac{\Sigma_{\mathrm{fs}}}{\sum_{\mathrm{s}}}\right]\left[\frac{\omega_{\mathrm{sf}}}{\omega_{\mathrm{ss}}}\right]=\left[\frac{\Sigma_{\mathrm{f}} \omega_{\mathrm{sf}}+\Sigma_{\mathrm{fs}} \omega_{\mathrm{ss}}}{\sum_{\mathrm{sf}} \omega_{\mathrm{sf}}+\Sigma_{\mathrm{s}} \omega_{\mathrm{ss}}}\right]=\lambda_{\mathrm{h}}\left[\frac{\omega_{\mathrm{sf}}}{\omega_{\mathrm{ss}}}\right] \\
& \Sigma_{\mathrm{f}} \omega_{\mathrm{sf}}+\Sigma_{\mathrm{fs}} \omega_{\mathrm{ss}}=\lambda_{\mathrm{h}} \omega_{\mathrm{sf}} \\
& \Sigma_{\mathrm{s}} \omega_{\mathrm{ss}}+\Sigma_{\mathrm{sf}} \omega_{\mathrm{sf}}=\lambda_{\mathrm{h}} \omega_{\mathrm{ss}}
\end{aligned}
$$

By comparing equation (3) with (13), (5) with (14), we will get inside of the difference between our approach and Tang and Wang's work. Equation (13) and (14) reveals there're interdependencies between $\omega_{\mathrm{sf}}$ and $\omega_{\mathrm{ss}}$. If we calculate $\omega_{\mathrm{sf}}$ and $\omega_{\text {ss }}$ independently, like (3) and (5), we will lose this interdependency in our model, and the representative power of our model will be degraded. By equation (13) and (14), we claim our approach is a better approach and will outperform the previous work.

\subsection{Recognition Stage}

After we successfully synthesize the original face image of the probe sketch, we would like to do recognition to identify who this person is. Unlike previous work, which simply uses Euclidean distance to compare the distance between the synthesized face image and the face images in database, we propose to use a frequency-based, illumination tolerant technique, which is advanced correlation filters. 

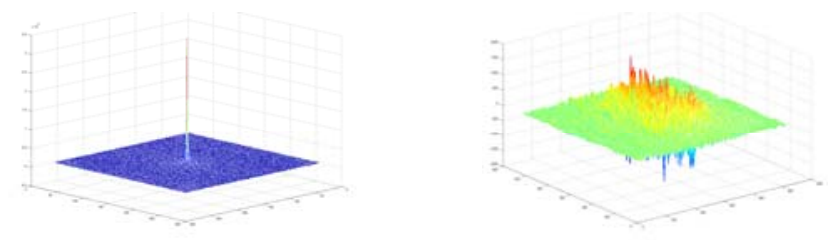

Figure 5: Left: correlation plane of an authentic sample. Right: Correlation plane of an imposter sample

Advanced correlation filters ${ }^{10}$ are advanced template-based classifiers that when correlated with an image result in a correlation plane. The correlation plane $\boldsymbol{C}$ measures the correlation between the filter and the image. Correlation of a class-specific filter with authentic and impostor data yield very different correlation planes. Fig. 5 demonstrates this difference. These advanced correlation filters optimize specific criteria to obtain sharp correlation peak outputs as shown below; this is very different from matched filters or normalized correlation approaches which are more common in the literature.

To quantify the difference between the two types of correlation planes, we define a measure of recognition called Peak to Correlation Energy (PCE). This is a measure the sharpness of the largest peak in the correlation output with respect to the rest of the correlation plane.

$$
\operatorname{PCE}(C)=\frac{\max (|C|)-\text { mean }(|C|)}{s t d \cdot \operatorname{dev}(|C|)}
$$

The Minimum Average Correlation Energy (MACE) Filter ${ }^{11}$ is designed to minimize the average energy $\boldsymbol{E}$ in the correlation plane or Average Correlation Energy (ACE). In the filter design for $h$ we also constrain the value of the correlation peak at the origin to be set to 1 . Assuming that we have a matrix X which contains the 2D Fourier transforms of training images along the columns, we can write the linear constraints as follows:

$$
\mathrm{X}^{+} \mathrm{h}=\mathrm{u}
$$

To achieve peak sharpness we must then also minimize correlation plane energy, thus we compute the average power spectrum of the face image which is vectorized and placed on the diagonal of matrix $\boldsymbol{D}$.

Our goal is to minimize $\boldsymbol{E}$ which is defined as:

$$
E=h^{+} D h
$$

where ${ }^{+}$denotes the conjugate transpose. The constrained minimization of equation (17) results in the MACE filter $\boldsymbol{h}_{\text {MACE. }}$

$$
h_{M A C E}=D^{-1} X\left(X^{+} D^{-1} X\right)^{-1} u
$$

where $\boldsymbol{u}$ is the constrained peak values (vector of ones).

The Unconstrained MACE (UMACE) Filter ${ }^{12}$ removes the constraint on the peak value. By removing this constraint, we may be able to find a better solution to the energy minimization. Instead, we try to maximize the average value of the peaks or Average Correlation Height (ACH). The closed form solution to the UMACE filter $\boldsymbol{h}_{\text {UMACE}}$ :

$$
\boldsymbol{h}_{U M A C E}=\boldsymbol{D}^{-1} \boldsymbol{m}
$$

where $\boldsymbol{m}$ is the average of the columns of $\boldsymbol{X}$. 
We will consider generalizations of the MACE and UMACE filters called the Optimal Tradeoff Synthetic Discriminant Function (OTSDF) filter ${ }^{13}$ and the Unconstrained OTSDF (UOTSDF) filter respectively. These generalized filters offer sharp correlation peaks and some noise tolerance. Given a desired proportion of peak sharpness to noise tolerance, the filter designs $\boldsymbol{h}_{\text {OTSDF }}$ and $\boldsymbol{h}_{\text {UOTSDF }}$ are:

$$
\begin{aligned}
& h_{\text {OTSDF }}=T^{-1} X\left(X^{+} T^{-1} X\right)^{-1} u \\
& h_{\text {UOTSDF }}=T^{-1} \mathrm{~m}
\end{aligned}
$$

where $\boldsymbol{T}$ is defined as:

$$
T=\alpha D+\sqrt{1-\alpha^{2}} C \text { given } 0 \leq \alpha \leq 1
$$

where $C$ is the assumed to be white noise power spectral density (so in this case $\mathrm{C}=\mathrm{I}$ the identity matrix). In this paper we use OTSDF (alpha $=0.99$ ) throughout all the recognition experiments.

\section{DATA}

The database we used in our experiment is CMU-PIE database ${ }^{14}$. The PIE database consists two datasets which we will refer to as Light (images captured with ambient background lighting) and NoLight(images captured without any background lighting on). Light database contains the pictures which were taken under sufficient environmental lighting, so in general one can see clear face images in all pictures in Light database. NoLight database contains pictures taken in the harshest illumination conditions, so the face suffers with larger cast shadows making face recognition in NoLight database a much harder task than in the PIE Light database because of these harsh illumination variations.

There are 65 people in both databases. In Light database, each person has 22 images; in NoLight, each person has 21 images captured under different lighting variations. Total number of images in this database is 2795. CMU-PIE database has following characteristics:

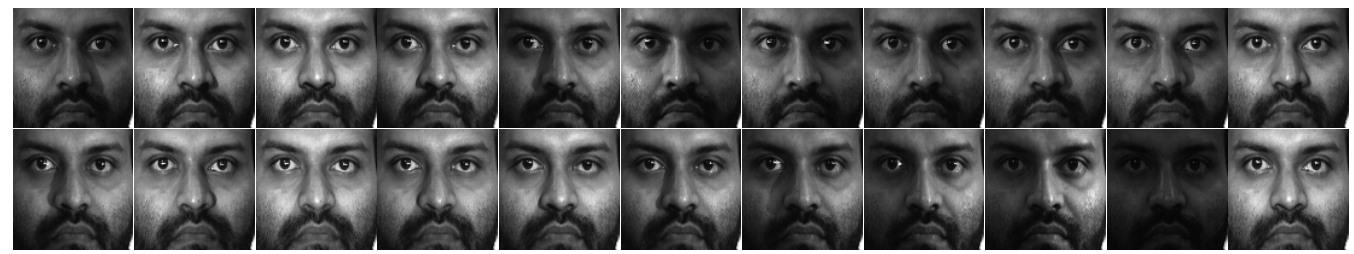

Figure 6: Examples of CMU-PIE-Light database. For each person there're 22 images, each of which has different orientation of illumination.

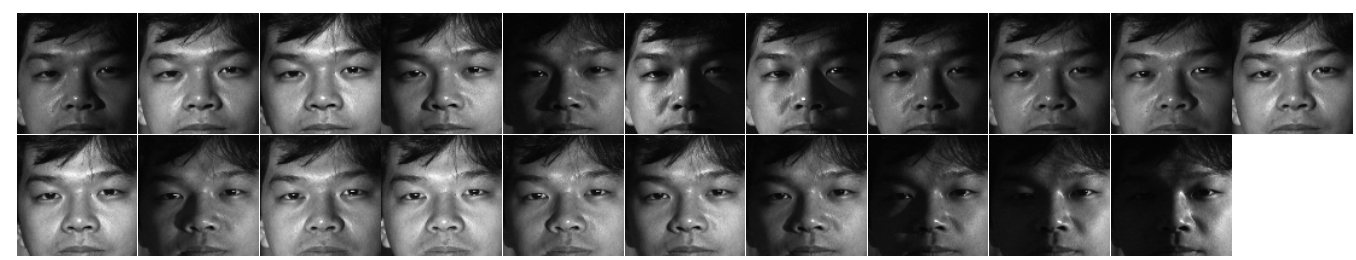

Figure 7: Examples of CMU-PIE-NoLight database. For each person there're 21 images, each of which has different orientation of illumination. 
- Contains both male and female faces

- Contains people from different race and color

- Contains images of people with and without glasses.

- Contains severe illumination variation across images of each person, as shown in Fig. 6 and 7.

As one can imagine, due to the large variation in gender, skin colour, the presence or absence of eye-glasses, and illumination; this is a very challenging task to perform face recognition on this database. Examples of face and their sketch counterpart images are shown in Fig. 1.

During the Training Stage, we selected two images with evenly distributed illumination (i.e. neutral frontal lighting) from every person, calculate eigenvectors in hybrid subspace. During recognition, we pick one sketch image, then synthesize the face image using algorithm described in Section 2.2. After building an OTSDF filter for this reconstructed face image, we use this filter to match all other images (all images except the two for training and the one for reconstruction). The rational for doing this is to simulate the real scenario when our system is applied in real life where the person we are looking for is walking under varying illumination causing their facial appearance to vary significantly due to lighting. The proposed method will allow us to match the reconstructed face images with those pictures taken from a surveillance camera. So we believe this experiment setting will yield results which are more strongly related with the one we would get in real world application.

\section{RESULTS}

In order to see the performance of the proposed method, we contrast it with $1 \mathrm{NN}$ classifier. Moreover, we also use different number of eigenfaces in Synthesis Stage to see if the proposed method degraded gracefully when the quality of synthesized images is getting worse. In addition, all the experiment results are based on the first rank, i.e: we only take the one with the highest score, both OTSDF and 1NN. Fig. 8 and 9 shows the result.

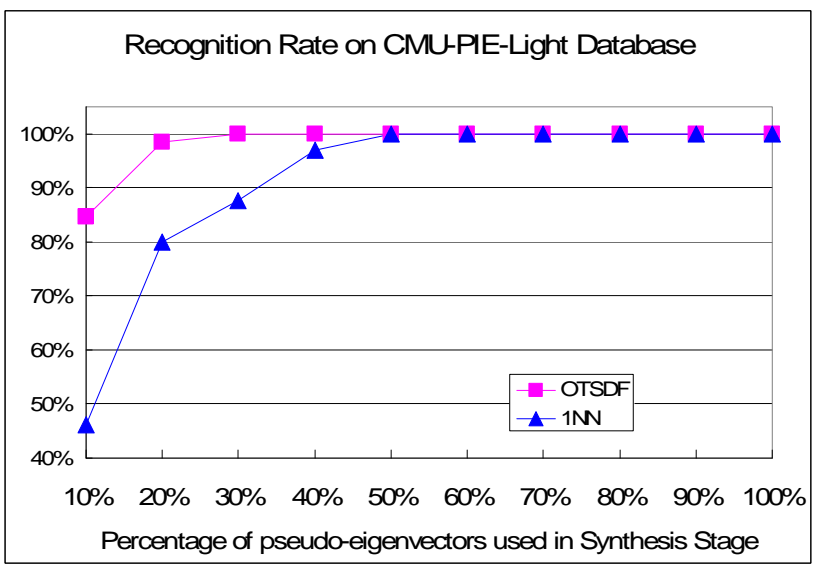

Figure 8: Recognition rate on CMU-PIE-Light database

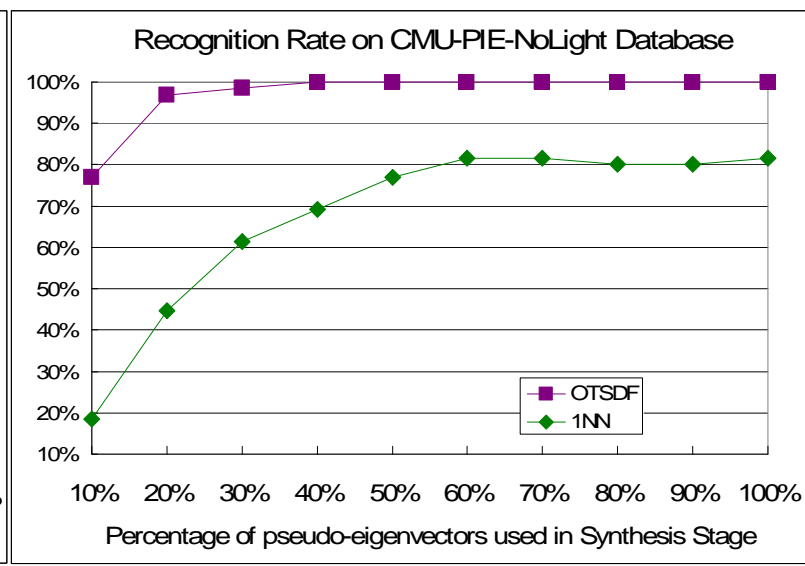

Figure 9: Recognition rate on CMU-PIE-NoLight database

\section{CONCLUSION}

The experimental results obtained are very encouraging. When experimenting on PIE Light database, which is a relatively easier task, we can get $100 \%$ recognition rate with either the OTSDF or 1NN method. However, OTSDF can achieve $100 \%$ even when only $30 \%$ of eigenvectors are used, while the $1 \mathrm{NN}$ can only achieve recognition rate of $87.69 \%$. 
When experimenting on the CMU PIE NoLight dataset, which is a much more challenging task, the OTSDF approach clearly outperformed $1 \mathrm{NN}$ in all experiments clearly showing its capabilities to perform illumination tolerant face recognition. From these experimental results, we can conclude that our proposed novel face synthesis from sketch approach coupled with advanced correlation filters for face recognition is a successful solution to this problem and is

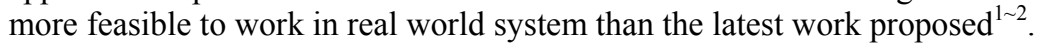

\section{FUTURE WORK}

We plan to apply our approach on larger dataset, for example, FERET ${ }^{15}$ and the Notre Dame Face Recognition Grand Challenge ${ }^{16}$ to see the performance of our proposed method in large scale face database.

\section{ACKNOWLEDGEMENT}

We would like to thank the U.S. Technical Support Working Group (TSWG) and for CMU CyLab for supporting this work.

\section{REFERENCE}

1. X. Tang and X. Wang, "Face Photo Recognition Using Sketch", Proc. of Int. Conf. Image Processing, 2002.

2. X. Tang and X. Wang, "Face Sketch Recognition", IEEE Trans. on Circuit System and Video Technology, Vol.14, pp.50-57, 2004.

3. X. Tang and X. Wang, "Face Sketch Synthesis and Recognition", Proc. of Int. Conf. Computer Vision, 2003.

4. Q. Liu, X. Tang, H, Jin, H. Lu, and S. Ma, "A Nonlinear Approach for Face Sketch Synthesis and Recognition", Proc. Of. IEEE Computer Society Conference on Computer Vision and Pattern Recognition, 2005.

5. M.A. Turk and A. P. Pentland, "Face recognition using eigenfaces", presented at Computer Vision and Pattern Recognition, 1991. Proceeding CVPR '91., IEEE Computer Society Conference on, 1991.

6. M. Savvides, B.V.K. Vijaya Kumar and P.K. Khosla, "Robust, Shift-Invariant Biometric Identification from Partial Face Images", Biometric Technologies for Human Identification (OR51) 2004.

7. M. Savvides, B.V.K. Vijaya Kumar and P.K. Khosla, "Face verification using correlation filters", Proc. Of the Third IEEE Automatic Identification Advanced Technologies, 56-61, Tarrytown, NY, March 2002.

8. M. Savvides, C.Xie, N. Chu, B.V.K.Vijaya Kumar, C. Podilchuk, A. Patel, A. Harthattu, R. Mammone, "Robust Face Recognition using Advanced Correlation Filters with Bijective-Mapping Preprocessing", Audio-Video Based Biometric Person Authentication (AVBPA), July 2005.

9. M.A. Turk and A. P. Pentland, "Face recognition using eigenfaces", presented at Computer Vision and Pattern Recognition, 1991. Proceeding CVPR '91., IEEE Computer Society Conference on, 1991.

10. B.V.K. Vijaya Kumar, "Tutorial Survey of Composite Filter designs for Optical Correlators," Applied Optics, vol. 31, pp. 4773-4801, 1992

11. A. Mahalanobis, B.V.K. Vijaya Kumar, and D. Casasent, "Minimum average correlation energy filters," Applied Optics, vol. 26:3633-3640, 1987.

12. A. Mahalanobis, B.V.K. Vijaya Kumar, S. Song, S.R.F. Sims, and J.F. Epperson, "Unconstrained correlation filters," Applied Optics, vol. 33:3751-3759, 1994.

13. B. V. K. V. Kumar, D. Carlson, and A. Mahalanobis, "Optimal tradeoff synthetic discriminant function (OTSDF) filters for arbitrary devices,” Opt. Lett., vol. 19, pp. 1556-1558, 1994.

14. T. Sim, S. Baker, and M. Bsat "The CMU Pose, Illumination, and Expression (PIE) Database of Human faces," Tech. Report CMU-RI-TR-01-02, Robotics Institute, Carnegie Mellon University, January (2001)

15. P. J. Phillips, H. Moon, P. J. Rauss, and S. Rizvi, "The FERET evaluation methodology for face recognition algorithms", IEEE Transactions on Pattern Analysis and Machine Intelligence, Vol. 22, No. 10, October 2000.

16. P. J. Phillips, "Face Recognition Grand Challenge", presented at Biometric Consortium Conference, 2004 